\title{
Exponential stabilization of uncertain nonlinear time-delay systems
}

Yali Dong ${ }^{1 *}$ and Jinying Liu $^{1,2}$

${ }^{*}$ Correspondence:

dongyl@vip.sina.com

'School of Science, Tianjin

Polytechnic University, Tianjin,

300387, China

Full list of author information is

available at the end of the article

\section{空 Springer}

\begin{abstract}
This paper is concerned with the problem of exponential stabilization for a class of uncertain nonlinear systems with state delay by means of periodically intermittent control. Based on the Lyapunov-Krasovskii functional approach, several sufficient conditions of exponential stabilization for this class of uncertain nonlinear systems with state delay are proposed, which have been expressed in terms of linear matrix inequalities (LMIs) whose feasibility can be easily checked by using the numerically efficient Matlab LMI Toolbox. Further, the control design method is extended to a class of nonlinear systems with state delay. And the new stability criterion is also presented, which guarantees the closed-loop systems are exponentially stable. Finally, two numerical examples are given to show the effectiveness of the proposed approach.
\end{abstract}

MSC: 93D15; 93C55; 34K20; 34D23

Keywords: time-delay nonlinear systems; periodically intermittent control; exponential stabilization; Lyapunov-Krasovskii functional; linear matrix inequality (LMI)

\section{Introduction}

Time delay naturally appears in many control systems and is frequently a source of instability [1-11]. In recent years, the problem of stability and stabilization of time-delay systems is a topic of great practical importance, which has attracted a lot of interest $[1-4,10-20]$. In [2], in the light of the Razumikhin stability theorem combined with the Newton-Leibniz formula, a new delay-dependent exponential stability condition was derived for linear non-autonomous time-delay systems. In [10], the sufficient conditions for absolute stability of nonlinear control systems of neutral type were obtained, and the estimates of exponential damping of solutions were constructed. In [12], the robust controller design for uncertain input-delayed systems was considered, and the stability criterion of the closedloop system was derived. In [16], stability results of quadratic discrete systems in the critical case were presented, and the stability domains were estimated. In [18], an improved delay-dependent stability condition for a system with multiple additive delay components was derived by using the Lyapunov second method. In [21], the sufficient conditions for global asymptotic stabilization of nonlinear systems were given, and the corresponding feedback control laws were designed. In [22], the problem of quadratic stabilization of multi-input multi-output switched nonlinear systems under an arbitrary switching law was investigated. The state feedbacks were designed, and a common quadratic Lyapunov function of all the closed-loop subsystems was constructed to realize quadratic stabilization of the class of switched nonlinear systems under an arbitrary switching law.

(c) 2012 Dong and Liu; licensee Springer. This is an Open Access article distributed under the terms of the Creative Commons Attribution License (http://creativecommons.org/licenses/by/2.0), which permits unrestricted use, distribution, and reproduction in any medium, provided the original work is properly cited. 
Recently, in-continuous control techniques such as impulsive control [23] and piecewise feedback control [24] have attracted much attention. In [23], impulsive control for master-slave synchronization schemes that consist of identical chaotic Lur'e systems was considered. Impulsive control laws which made use of linear static measurement feedback instead of full state feedback were investigated. The recent paper [24] studied the output regulation problem for a class of discrete-time nonlinear systems under periodic disturbances generated from the so-called exosystems. By exploiting the structural information encoded in the fuzzy rules, a piecewise state-feedback and a piecewise error-feedback control laws were constructed to achieve asymptotic rejecting of the unwanted disturbances and/or tracking of the desired trajectories.

In this paper, we investigate the problem of exponential stabilization for a class of uncertain nonlinear systems by using another in-continuous feedback, i.e., periodically intermittent control. Intermittent control is a special form of switching control [25]. It has been used for a variety of purposes in engineering fields such as manufacturing, transportation, air-quality control, communication and so on. Recently, intermittent control has been introduced to chaotic dynamical systems [26-28]. [26] described the method of synchronizing slave to the master trajectory using intermittent coupling. However, [26] gave little theoretical analysis for intermittent control systems, but only many numerical simulations. In [27], the authors investigated the exponential stabilization problem for a class of chaotic systems with delay by means of periodically intermittent control. In [28], the quasi-synchronization problem for chaotic neural networks with parameter mismatch was formulated via periodically intermittent control.

Motivated by the aforementioned discussion, in this paper, we study a class of intermittent control with time duration for a class of uncertain nonlinear time-delay systems. Namely, the control is activated in certain nonzero time intervals, and it is off in other time intervals. Based on the Lyapunov-Krasovskii functional approach, exponential stability criteria for the class of uncertain nonlinear time-delay systems are given, which are presented in terms of linear matrix inequalities (LMIs). Moreover, this approach of control design has been generalized to a class of nonlinear systems with state delay. The new exponentially stabilization criterion for this class of nonlinear time-delay systems is proposed. Finally, two numerical examples are given to demonstrate the validity of the result.

The rest of this paper is organized as follows. In Section 2, the intermittent control problem is formulated and some notations and lemmas are introduced. In Section 3, the exponential stabilization problem for a class of uncertain nonlinear time-delay systems is investigated by means of periodically intermittent control, and some exponential stability criterions are established. Furthermore, the exponential stabilization method is expanded to a class of nonlinear systems with state delay. Two numerical examples are presented in Section 4. Finally, some conclusions and remarks are drawn in Section 5.

\section{Problem formulation and preliminaries}

Consider a class of nonlinear systems with delayed state described by

$$
\begin{aligned}
\dot{x}(t)= & (A+\Delta A(t)) x(t)+\left(A_{d}+\Delta A_{d}(t)\right) x(t-d) \\
& +(B+\Delta B(t)) u(t)+f(x(t), x(t-d)), \\
x(t)= & \phi(t), \quad t \in[-d, 0],
\end{aligned}
$$


where $x \in R^{n}$ is a state vector, and $u \in R^{m}$ is the external input of the system (2.1). $f: R^{n} \rightarrow R^{n}$ is a continuous nonlinear function with $f(0,0)=0$, and there exist positive definite matrices $Q_{1}, Q_{2}$ such that $\|f(x(t), x(t-d))\|^{2} \leq x^{T}(t) Q_{1} x(t)+x^{T}(t-d) Q_{2} x(t-d)$ for $x(t), x(t-d) \in R^{n}$. The initial function $\phi(t) \in C\left([-d, 0], R^{n}\right), d>0$, has its norm $\|\phi\|=$ $\sup _{s \in[-d, 0]}\|\phi(s)\| . \Delta A(t), \Delta A_{d}(t)$ and $\Delta B(t)$ are time-varying uncertainties, which satisfy the following conditions:

$$
\Delta A(t)=D_{1} F(t) E_{1}, \quad \Delta A_{d}(t)=D_{2} F(t) E_{2}, \quad \Delta B(t)=D_{3} F(t) E_{3},
$$

where $D_{i}, E_{i}, i=1,2,3$, are real constant matrices of appropriate dimensions, and $F(t)$ is an unknown time-varying matrix with $F^{T}(t) F(t) \leq I$.

The following lemmas are useful in the proof of our main results.

Lemma 2.1 ([29]) Let D, E and $F$ be real matrices of appropriate dimensions with $F^{T} F \leq I$, then for any scalar $\varepsilon>0$, we have the following inequality:

$$
D F E+E^{T} F^{T} D^{T} \leq \varepsilon^{-1} D D^{T}+\varepsilon E^{T} E .
$$

Lemma 2.2 ([30]) Let $M, N$ be real matrices of appropriate dimensions. Then for any matrix $Q>0$ of appropriate dimension and any scalar $\beta>0$, the following inequality holds:

$$
M N+N^{T} M^{T} \leq \beta^{-1} M Q^{-1} M^{T}+\beta N^{T} Q N .
$$

Lemma 2.3 ([31]) Given constant matrices $S_{1}, S_{2}$ and $S_{3}$ with appropriate dimensions, where $S_{1}=S_{1}^{T}$, and $0<S_{3}=S_{3}^{T}$, then $S_{1}+S_{2} S_{3}^{-1} S_{2}^{T}<0$ if and only if

$$
\left[\begin{array}{cc}
S_{1} & S_{2} \\
S_{2}^{T} & -S_{3}
\end{array}\right]<0
$$

In order to stabilize the system (2.1) by means of periodically intermittent feedback control, we assume that the control imposed on the system is of the form

$$
u(t)= \begin{cases}K x(t), & n T \leq t<n T+\tau, \\ 0, & n T+\tau \leq t<(n+1) T,\end{cases}
$$

where $K \in R^{m \times n}$ is the control gain matrix, $T>0$ denotes the control period, and $\tau>0$ is called the control width.

With the control law (2.3), the system (2.1) can be rewritten as

$$
\left\{\begin{array}{rlrl}
\dot{x}(t)= & (A+\Delta A(t)) x(t)+\left(A_{d}+\Delta A_{d}(t)\right) x(t-d) & & \\
& +(B+\Delta B(t)) K x(t)+f(x(t), x(t-d)), & & n T \leq t<n T+\tau, \\
\dot{x}(t)= & (A+\Delta A(t)) x(t)+\left(A_{d}+\Delta A_{d}(t)\right) x(t-d) & & \\
& +f(x(t), x(t-d)), & n T+\tau \leq t<(n+1) T .
\end{array}\right.
$$

This is a classical uncertain switched system with delayed state where the switching rule only depends on the time. Although there are many successful applications of intermit- 
tent control, the theoretical analysis of an intermittent control system has received little attention. In this brief, we will make a contribution to this issue.

Throughout this brief, we use $P^{T}, \lambda_{\min }(P)\left(\lambda_{\max }(P)\right)$ to denote the transpose and the minimum (maximum) eigenvalue of a square matrix $P$, respectively. The vector (or matrix) norm is taken to be Euclidean, denoted by $\|\cdot\|$. We use $P>0(<0, \leq 0, \geq 0)$ to denote a positive (negative, semi-negative, semi-positive) definite matrix $P$. The symmetric term in a symmetric matrix is denoted as $*$.

\section{Exponential stabilization of a class of nonlinear systems with time-delay}

This section addresses the exponential stability problem of the switched system (2.4). The main result is stated as follows.

Theorem 3.1 If there exist positive definite matrices $P>0, Q>0$ and scalar constants $\varepsilon_{i j}>0(i=1,2, j=1,2,3), \varepsilon_{14}>0, w>0$, such that the following matrix inequalities hold:

$$
\begin{aligned}
& {\left[\begin{array}{cc}
\Omega_{1} & P A_{d} \\
A_{d}^{T} P & \varepsilon_{11}^{-1} Q_{2}+\varepsilon_{13} E_{2}^{T} E_{2}-e^{-w d} Q
\end{array}\right]<0,} \\
& {\left[\begin{array}{cc}
\Omega_{2} & P A_{d} \\
A_{d}^{T} P & \varepsilon_{21}^{-1} Q_{2}+\varepsilon_{23} E_{2}^{T} E_{2}-e^{-w d} Q
\end{array}\right]<0,}
\end{aligned}
$$

where

$$
\begin{aligned}
\Omega_{1}= & A^{T} P+P A+K^{T} B^{T} P+P B K+w P+Q+\varepsilon_{11} P P+\varepsilon_{11}^{-1} Q_{1}+\varepsilon_{12}^{-1} E_{1}^{T} E_{1}+\varepsilon_{12} P D_{1} D_{1}^{T} P \\
& +\varepsilon_{14}^{-1} K^{T} E_{3}^{T} E_{3} K+\varepsilon_{14} P D_{3} D_{3}^{T} P+\varepsilon_{13}^{-1} P D_{2} D_{2}^{T} P,
\end{aligned}
$$

and

$$
\Omega_{2}=A^{T} P+P A+w P+Q+\varepsilon_{21} P P+\varepsilon_{21}^{-1} Q_{1}+\varepsilon_{22}^{-1} E_{1}^{T} E_{1}+\varepsilon_{22} P D_{1} D_{1}^{T} P+\varepsilon_{23}^{-1} P D_{2} D_{2}^{T} P
$$

then the system (2.4) is exponentially stable. Moreover, the solution $x(t)$ satisfies the condition

$$
\|x(t)\| \leq\|\phi\| \sqrt{\frac{\lambda_{\max }(P)+d \lambda_{\max }(Q)}{\lambda_{\min }(P)}} e^{-\frac{w(t-\tau)}{2}}, \quad \forall t>0 .
$$

Proof Consider the following candidate Lyapunov-Krasovskii functional:

$$
V(x(t))=x^{T}(t) P x(t)+e^{-w t} \int_{t-d}^{t} e^{w \theta} x^{T}(\theta) Q x(\theta) d \theta .
$$

When $n T \leq t<n T+\tau$, the derivative of (3.3) with respect to time $t$ along the trajectories of the first subsystem of the system (2.4) is calculated and estimated as follows:

$$
\begin{aligned}
\dot{V}(x(t))= & \dot{x}^{T}(t) P x(t)+x^{T}(t) P \dot{x}(t)-w e^{-w t} \int_{t-d}^{t} e^{w \theta} x^{T}(\theta) Q x(\theta) d \theta \\
& +e^{-w t}\left[e^{w t} x^{T}(t) Q x(t)-e^{w(t-d)} x^{T}(t-d) Q x(t-d)\right]
\end{aligned}
$$




$$
\begin{aligned}
= & \dot{x}^{T}(t) P x(t)+x^{T}(t) P \dot{x}(t)-w e^{-w t} \int_{t-d}^{t} e^{w \theta} x^{T}(\theta) Q x(\theta) d \theta \\
& +x^{T}(t) Q x(t)-e^{-w d} x^{T}(t-d) Q x(t-d) \\
= & {\left[(A+\Delta A(t)) x(t)+\left(A_{d}+\Delta A_{d}(t)\right) x(t-d)\right.} \\
& +(B+\Delta B(t)) K x(t)+f(x(t), x(t-d))]^{T} P x(t) \\
& +x^{T}(t) P\left[(A+\Delta A(t)) x(t)+\left(A_{d}+\Delta A_{d}(t)\right) x(t-d)\right. \\
& +(B+\Delta B(t)) K x(t)+f(x(t), x(t-d))] \\
& -w e^{-w t} \int_{t-d}^{t} e^{w \theta} x^{T}(\theta) Q x(\theta) d \theta+x^{T}(t) Q x(t)-e^{-w d} x^{T}(t-d) Q x(t-d) \\
= & x^{T}(t)\left[A^{T} P+P A+K^{T} B^{T} P+P B K+Q\right] x(t) \\
& +x^{T}(t-d) A_{d}^{T} P x(t)+x^{T}(t) P A_{d} x(t-d) \\
& +2 x^{T}(t) P f(x(t), x(t-d))+x^{T}(t)\left[\Delta A^{T}(t) P+P \Delta A(t)\right. \\
& \left.+K^{T} \Delta B^{T}(t) P+P \Delta B(t) K\right] x(t) \\
& +x^{T}(t-d) \Delta A_{d}^{T}(t) P x(t)+x^{T}(t) P \Delta A_{d}(t) x(t-d) \\
& -w V(x(t))+w x^{T}(t) P x(t)-e^{-w d} x^{T}(t-d) Q x(t-d) \\
\leq & x^{T}(t)\left[A^{T} P+P A+K^{T} B^{T} P+P B K+w P+Q\right] x(t) \\
& +x^{T}(t-d) A_{d}^{T} P x(t)+x^{T}(t) P A_{d} x(t-d)+2 x^{T}(t) P f(x(t), x(t-d)) \\
& +x^{T}(t)\left[E_{1}^{T} F^{T}(t) D_{1}^{T} P+P D_{1} F(t) E_{1}+K^{T} E_{3}^{T} F^{T}(t) D_{3}^{T} P\right. \\
& \left.+P D_{3} F(t) E_{3} K\right] x(t)+x^{T}(t-d) E_{2}^{T} F^{T}(t) D_{2}^{T} P x(t)+x^{T}(t) P D_{2} F(t) E_{2} x(t-d) \\
& -w V(x(t))-e^{-w d} x^{T}(t-d) Q x(t-d) . \\
& +w^{T}(t) \\
&
\end{aligned}
$$

Using Lemma 2.1 and Lemma 2.2, we get

$$
\begin{aligned}
\dot{V}(x(t)) \leq & x^{T}(t)\left[A^{T} P+P A+K^{T} B^{T} P+P B K+w P+Q\right] x(t) \\
& +x^{T}(t-d) A_{d}^{T} P x(t)+x^{T}(t) P A_{d} x(t-d) \\
& +\varepsilon_{11} x^{T}(t) P P x(t)+\varepsilon_{11}^{-1}\|f(x(t), x(t-d))\|^{2} \\
& +x^{T}(t)\left[\varepsilon_{12}^{-1} E_{1}^{T} E_{1}+\varepsilon_{12} P D_{1} D_{1}^{T} P+\varepsilon_{14}^{-1} K^{T} E_{3}^{T} E_{3} K\right. \\
& \left.+\varepsilon_{14} P D_{3} D_{3}^{T} P\right] x(t)+\varepsilon_{13} x^{T}(t-d) E_{2}^{T} E_{2} x(t-d) \\
& +\varepsilon_{13}^{-1} x^{T}(t) P D_{2} D_{2}^{T} P x(t)-w V(x(t)) \\
& -e^{-w d} x^{T}(t-d) Q x(t-d) \\
\leq & x^{T}(t)\left[A^{T} P+P A+K^{T} B^{T} P+P B K+w P+Q\right] x(t) \\
& +x^{T}(t-d) A_{d}^{T} P x(t)+x^{T}(t) P A_{d} x(t-d) \\
& +\varepsilon_{11} x^{T}(t) P P x(t)+\varepsilon_{11}^{-1}\left[x^{T}(t) Q_{1} x(t)+x^{T}(t-d) Q_{2} x(t-d)\right] \\
& +x^{T}(t)\left[\varepsilon_{12}^{-1} E_{1}^{T} E_{1}+\varepsilon_{12} P D_{1} D_{1}^{T} P+\varepsilon_{14}^{-1} K^{T} E_{3}^{T} E_{3} K+\varepsilon_{14} P D_{3} D_{3}^{T} P\right] x(t) \\
& +\varepsilon_{13}^{-1} x^{T}(t) P D_{2} D_{2}^{T} P x(t)+\varepsilon_{13} x^{T}(t-d) E_{2}^{T} E_{2} x(t-d)
\end{aligned}
$$




$$
\begin{aligned}
& -w V(x(t))-e^{-w d} x^{T}(t-d) Q x(t-d) \\
= & x^{T}(t)\left[A^{T} P+P A+K^{T} B^{T} P+P B K+w P+Q\right. \\
& +\varepsilon_{11} P P+\varepsilon_{11}^{-1} Q_{1}+\varepsilon_{12}^{-1} E_{1}^{T} E_{1}+\varepsilon_{12} P D_{1} D_{1}^{T} P \\
& \left.+\varepsilon_{14}^{-1} K^{T} E_{3}^{T} E_{3} K+\varepsilon_{14} P D_{3} D_{3}^{T} P+\varepsilon_{13}^{-1} P D_{2} D_{2}^{T} P\right] x(t) \\
& +x^{T}(t-d) A_{d}^{T} P x(t)+x^{T}(t) P A_{d} x(t-d) \\
& +x^{T}(t-d)\left[\varepsilon_{11}^{-1} Q_{2}+\varepsilon_{13} E_{2}^{T} E_{2}-e^{-w d} Q\right] x(t-d)-w V(x(t)) \\
= & {\left[\begin{array}{c}
x(t) \\
x(t-d)
\end{array}\right]^{T}\left[\begin{array}{cc}
\Omega_{1} & P A_{d} \\
A_{d}^{T} P & \varepsilon_{11}^{-1} Q_{2}+\varepsilon_{13} E_{2}^{T} E_{2}-e^{-w d} Q
\end{array}\right]\left[\begin{array}{c}
x(t) \\
x(t-d)
\end{array}\right]-w V(x(t)), }
\end{aligned}
$$

where

$$
\begin{aligned}
\Omega_{1}= & A^{T} P+P A+K^{T} B^{T} P+P B K+w P+Q+\varepsilon_{11} P P+\varepsilon_{11}^{-1} Q_{1}+\varepsilon_{12}^{-1} E_{1}^{T} E_{1}+\varepsilon_{12} P D_{1} D_{1}^{T} P \\
& +\varepsilon_{14}^{-1} K^{T} E_{3}^{T} E_{3} K+\varepsilon_{14} P D_{3} D_{3}^{T} P+\varepsilon_{13}^{-1} P D_{2} D_{2}^{T} P .
\end{aligned}
$$

From (3.1), we have

$$
\dot{V}(x(t)) \leq-w V(x(t)), \quad n T \leq t<n T+\tau,
$$

which implies that when $n T \leq t<n T+\tau$,

$$
V(x(t)) \leq V(x(n T)) e^{-w(t-n T)} .
$$

Similarly, when $n T+\tau \leq t<(n+1) T$, we have

$$
\begin{aligned}
\dot{V}(x(t))= & \dot{x}^{T}(t) P x(t)+x^{T}(t) P \dot{x}(t)-w e^{-w t} \int_{t-d}^{t} e^{w \theta} x^{T}(\theta) Q x(\theta) d \theta \\
& +x^{T}(t) Q x(t)-e^{-w d} x^{T}(t-d) Q x(t-d) \\
= & {\left[(A+\Delta A(t)) x(t)+\left(A_{d}+\Delta A_{d}(t)\right) x(t-d)+f(x(t), x(t-d))\right]^{T} P x(t) } \\
& +x^{T}(t) P\left[(A+\Delta A(t)) x(t)+\left(A_{d}+\Delta A_{d}(t)\right) x(t-d)+f(x(t), x(t-d))\right] \\
& -w e^{-w t} \int_{t-d}^{t} e^{w \theta} x^{T}(\theta) Q x(\theta) d \theta+x^{T}(t) Q x(t)-e^{-w d} x^{T}(t-d) Q x(t-d) \\
= & x^{T}(t)\left[A^{T} P+P A+Q\right] x(t)+x^{T}(t-d) A_{d}^{T} P x(t)+x^{T}(t) P A_{d} x(t-d) \\
& +2 x^{T}(t) P f(x(t), x(t-d))+x^{T}(t)\left[\Delta A^{T}(t) P+P \Delta A(t)\right] x(t) \\
& +x^{T}(t-d) \Delta A_{d}^{T}(t) P x(t)+x^{T}(t) P \Delta A_{d}(t) x(t-d) \\
& -w V(x(t))+w x^{T}(t) P x(t)-e^{-w d} x^{T}(t-\tau) Q x(t-\tau) \\
\leq & x^{T}(t)\left[A^{T} P+P A+w P+Q\right] x(t)+x^{T}(t-d) A_{d}^{T} P x(t)+x^{T}(t) P A_{d} x(t-d) \\
& +2 x^{T}(t) P f(x(t), x(t-d))+x^{T}(t)\left[E_{1}^{T} F^{T}(t) D_{1}^{T} P+P D_{1} F(t) E_{1}\right] x(t) \\
& +x^{T}(t-d) E_{2}^{T} F^{T}(t) D_{2}^{T} P x(t)+x^{T}(t) P D_{2} F(t) E_{2} x(t-d) \\
& -w V(x(t))-e^{-w d} x^{T}(t-d) Q x(t-d)
\end{aligned}
$$




$$
\begin{aligned}
\leq & x^{T}(t)\left[A^{T} P+P A+w P+Q\right] x(t)+x^{T}(t-d) A_{d}^{T} P x(t)+x^{T}(t) P A_{d} x(t-d) \\
& +\varepsilon_{21} x^{T}(t) P P x(t)+\varepsilon_{21}^{-1}\|f(x(t), x(t-d))\|^{2} \\
& +x^{T}(t)\left[\varepsilon_{22}^{-1} E_{1}^{T} E_{1}+\varepsilon_{22} P D_{1} D_{1}^{T} P\right] x(t) \\
& +\varepsilon_{23} x^{T}(t-d) E_{2}^{T} E_{2} x(t-d)+\varepsilon_{23}^{-1} x^{T}(t) P D_{2} D_{2}^{T} P x(t)-w V(x(t)) \\
& -e^{-w d} x^{T}(t-d) Q x(t-d) \\
\leq & x^{T}(t)\left[A^{T} P+P A+w P+Q\right] x(t)+x^{T}(t-d) A_{d}^{T} P x(t)+x^{T}(t) P A_{d} x(t-d) \\
& +\varepsilon_{21} x^{T}(t) P P x(t)+\varepsilon_{21}^{-1}\left[x^{T}(t) Q_{1} x(t)+x^{T}(t-d) Q_{2} x(t-d)\right] \\
& +\varepsilon_{23}^{-1} x^{T}(t) P D_{2} D_{2}^{T} P x(t)+x^{T}(t)\left[\varepsilon_{22}^{-1} E_{1}^{T} E_{1}+\varepsilon_{22} P D_{1} D_{1}^{T} P\right] x(t) \\
& +\varepsilon_{23} x^{T}(t-d) E_{2}^{T} E_{2} x(t-d)-w V(x(t)) \\
& -e^{-w d} x^{T}(t-d) Q x(t-d) \\
= & x^{T}(t)\left[A^{T} P+P A+w P+Q+\varepsilon_{21} P P+\varepsilon_{21}^{-1} Q_{1}\right. \\
& \left.+\varepsilon_{22}^{-1} E_{1}^{T} E_{1}+\varepsilon_{22} P D_{1} D_{1}^{T} P+\varepsilon_{23}^{-1} P D_{2} D_{2}^{T} P\right] x(t) \\
& +x^{T}(t-d)\left[\varepsilon_{21}^{-1} Q_{2}+\varepsilon_{23} E_{2}^{T} E_{2}-e^{-w d} Q\right] x(t-d)-w V(x(t)) \\
& +x^{T}(t-d) A_{d}^{T} P x(t)+x^{T}(t) P A_{d} x(t-d) \\
= & {\left[\begin{array}{c}
x(t) \\
x(t-d)
\end{array}\right]^{T}\left[\begin{array}{cc}
\Omega_{2} & P A_{d} \\
A_{d}^{T} P & \varepsilon_{21}^{-1} Q_{2}+\varepsilon_{23} E_{2}^{T} E_{2}-e^{-w d} Q
\end{array}\right]\left[\begin{array}{c}
x(t) \\
x(t-d)
\end{array}\right]-w V(x(t)), }
\end{aligned}
$$

where $\Omega_{2}=A^{T} P+P A+w P+Q+\varepsilon_{21} P P+\varepsilon_{21}^{-1} Q_{1}+\varepsilon_{22}^{-1} E_{1}^{T} E_{1}+\varepsilon_{22} P D_{1} D_{1}^{T} P+\varepsilon_{23}^{-1} P D_{2} D_{2}^{T} P$.

From (3.2), we have

$$
\dot{V}(x(t)) \leq-w V(x(t)), \quad n T+\tau \leq t<(n+1) T,
$$

which implies that when $n T+\tau \leq t<(n+1) T$,

$$
V(x(t)) \leq V(x(n T+\tau)) e^{-w(t-n T-\tau)} .
$$

From (3.5) and (3.7), it follows that:

When $0 \leq t<\tau, V(x(t)) \leq V\left(x_{0}\right) e^{-w t}$, and

$$
V(x(\tau)) \leq V\left(x_{0}\right) e^{-w \tau} .
$$

When $\tau \leq t<T$,

$$
\begin{aligned}
V(x(t)) & \leq V(x(\tau)) e^{-w(t-\tau)} \\
& \leq V\left(x_{0}\right) e^{-w \tau} e^{-w(t-\tau)} \\
& =V\left(x_{0}\right) e^{-w t}, \\
V(x(T)) & \leq V\left(x_{0}\right) e^{-w T} .
\end{aligned}
$$


When $T \leq t<T+\tau$,

$$
\begin{aligned}
V(x(t)) & \leq V(x(T)) e^{-w(t-T)} \\
& \leq V\left(x_{0}\right) e^{-w T} e^{-w(t-T)} \\
& =V\left(x_{0}\right) e^{-w t}, \\
V(x(T+\tau)) & \leq V\left(x_{0}\right) e^{-w(T+\tau)} .
\end{aligned}
$$

When $T+\tau \leq t<2 T$,

$$
\begin{aligned}
V(x(t)) & \leq V(x(T+\tau)) e^{-w(t-T-\tau)} \\
& \leq V\left(x_{0}\right) e^{-w(\tau+T)} e^{-w(t-T-\tau)} \\
& =V\left(x_{0}\right) e^{-w t}, \\
V(x(2 T)) & \leq V\left(x_{0}\right) e^{-2 w T} .
\end{aligned}
$$

When $2 T \leq t<2 T+\tau$,

$$
\begin{aligned}
V(x(t)) & \leq V(x(2 T)) e^{-w(t-2 T)} \\
& \leq V\left(x_{0}\right) e^{-w 2 T} e^{-w(t-2 T)} \\
& =V\left(x_{0}\right) e^{-w t}, \\
V(x(2 T & +\tau)) \leq V\left(x_{0}\right) e^{-w(2 T+\tau)} .
\end{aligned}
$$

When $n T \leq t<n T+\tau$,

$$
\begin{aligned}
V(x(t)) & \leq V(x(n T)) e^{-w(t-n T)} \\
& \leq V\left(x_{0}\right) e^{-n w T} e^{-w(t-n T)} \\
& \leq V\left(x_{0}\right) e^{-w t} \\
& \leq V\left(x_{0}\right) e^{-w(t-\tau)} \\
V(x(n T+\tau)) & \leq V\left(x_{0}\right) e^{-w(n T)} .
\end{aligned}
$$

When $n T+\tau \leq t<(n+1) T$,

$$
\begin{aligned}
V(x(t)) & \leq V(x(n T+\tau)) e^{-w(t-n T-\tau)} \\
& \leq V\left(x_{0}\right) e^{-w n T} e^{-w(t-n T-\tau)} \\
& =V\left(x_{0}\right) e^{-w(t-\tau)} .
\end{aligned}
$$

From (3.8) and (3.9), it follows that for any $t>0$, we can obtain

$$
V(x(t)) \leq V\left(x_{0}\right) e^{-w(t-\tau)}, \quad \forall t>0 .
$$


From (3.3) and (3.10), it follows that for any $t>0$,

$$
\begin{aligned}
x^{T}(t) x(t) & \leq \frac{V(x(t))}{\lambda_{\min }(P)} \\
& \leq \frac{1}{\lambda_{\min }(P)} V\left(x_{0}\right) e^{-w(t-\tau)} .
\end{aligned}
$$

Hence, we get

$$
\|x(t)\| \leq \sqrt{\frac{V\left(x_{0}\right)}{\lambda_{\min }(P)}} e^{-\frac{w(t-\tau)}{2}}, \quad \forall t>0,
$$

Noticing

$$
\begin{aligned}
V\left(x_{0}\right) & =x^{T}(0) P x(0)+\int_{-d}^{0} e^{w \theta} x^{T}(\theta) Q x(\theta) d \theta \\
& \leq\left(\lambda_{\max }(P)+d \lambda_{\max }(Q)\right)\|\phi\|^{2},
\end{aligned}
$$

we get

$$
\|x(t)\| \leq\|\phi\| \sqrt{\frac{\lambda_{\max }(P)+d \lambda_{\max }(Q)}{\lambda_{\min }(P)}} e^{-\frac{w(t-\tau)}{2}}, \quad \forall t>0,
$$

which completes the proof.

Corollary 3.1 If there exist positive definite matrices $P>0, Q>0$, and scalar constants $\varepsilon_{j}>0(j=1,2,3,4), w>0$, such that the following LMIs hold:

$$
\left[\begin{array}{ccc}
\Pi_{1} & P A_{d} & P D_{3} \\
A_{d}^{T} P & \varepsilon_{1}^{-1} Q_{2}+\varepsilon_{3} E_{2}^{T} E_{2}-e^{-w d} Q & 0 \\
D_{3}^{T} P & 0 & -\varepsilon_{4}^{-1} I
\end{array}\right]<0,
$$

$$
\left[\begin{array}{ccccc}
\Pi_{2} & P A_{d} & P & P D_{1} & P D_{2} \\
P A_{d} & \varepsilon_{1}^{-1} Q_{2}+\varepsilon_{3} E_{2}^{T} E_{2}-e^{-w d} Q & 0 & 0 & 0 \\
P & 0 & -\varepsilon_{1}^{-1} I & 0 & 0 \\
D_{1}^{T} P & 0 & 0 & -\varepsilon_{2}^{-1} I & 0 \\
D_{2}^{T} P & 0 & 0 & 0 & -\varepsilon_{3}^{-1} I
\end{array}\right]<0,
$$

were

$$
\begin{aligned}
& \Pi_{1}=K^{T} B^{T} P+P B K+\varepsilon_{4}^{-1} K^{T} E_{3}^{T} E_{3} K, \\
& \Pi_{2}=A^{T} P+P A+w P+Q+\varepsilon_{1}^{-1} Q_{1}+\varepsilon_{2}^{-1} E_{1}^{T} E_{1},
\end{aligned}
$$

then the system (2.4) is exponentially stable. Moreover, the solution $x(t)$ satisfies the condition

$$
\|x(t)\| \leq\|\phi\| \sqrt{\frac{\lambda_{\max }(P)+d \lambda_{\max }(Q)}{\lambda_{\min }(P)}} e^{-\frac{w(t-\tau)}{2}}, \quad \forall t>0 .
$$


Proof Set

$$
\begin{aligned}
& \Omega_{3}=K^{T} B^{T} P+P B K+\varepsilon_{4}^{-1} K^{T} E_{3}^{T} E_{3} K+\varepsilon_{4} P D_{3} D_{3}^{T} P \\
& \Omega_{4}=A^{T} P+P A+w P+Q+\varepsilon_{1} P P+\varepsilon_{1}^{-1} Q_{1}+\varepsilon_{2}^{-1} E_{1}^{T} E_{1}+\varepsilon_{2} P D_{1} D_{1}^{T} P+\varepsilon_{3}^{-1} P D_{2} D_{2}^{T} P .
\end{aligned}
$$

According to Lemma 2.3 and (3.11) and (3.12), we have

$$
\begin{aligned}
& {\left[\begin{array}{cc}
\Omega_{3} & P A_{d} \\
A_{d}^{T} P & \varepsilon_{1}^{-1} Q_{2}+\varepsilon_{3} E_{2}^{T} E_{2}-e^{-w d} Q
\end{array}\right]<0,} \\
& {\left[\begin{array}{cc}
\Omega_{4} & P A_{d} \\
A_{d}^{T} P & \varepsilon_{1}^{-1} Q_{2}+\varepsilon_{3} E_{2}^{T} E_{2}-e^{-w d} Q
\end{array}\right]<0 .}
\end{aligned}
$$

Taking $\varepsilon_{11}=\varepsilon_{21}=\varepsilon_{1}, \varepsilon_{12}=\varepsilon_{22}=\varepsilon_{2}, \varepsilon_{13}=\varepsilon_{23}=\varepsilon_{3}, \varepsilon_{14}=\varepsilon_{4}$, we have

$$
\begin{aligned}
\Omega_{2} & =A^{T} P+P A+w P+Q+\varepsilon_{21} P P+\varepsilon_{21}^{-1} Q_{1}+\varepsilon_{22}^{-1} E_{1}^{T} E_{1}+\varepsilon_{22} P D_{1} D_{1}^{T} P+\varepsilon_{23}^{-1} P D_{2} D_{2}^{T} P \\
& =A^{T} P+P A+w P+Q+\varepsilon_{1} P P+\varepsilon_{1}^{-1} Q_{1}+\varepsilon_{2}^{-1} E_{1}^{T} E_{1}+\varepsilon_{2} P D_{1} D_{1}^{T} P+\varepsilon_{3}^{-1} P D_{2} D_{2}^{T} P \\
& =\Omega_{4},
\end{aligned}
$$

Hence, (3.14) implies that (3.2) holds and $\Omega_{4}<0$. From (3.13), we have

$$
\left[\begin{array}{cc}
\Omega_{3} & P A_{d} \\
A_{d}^{T} P & \varepsilon_{1}^{-1} Q_{2}+\varepsilon_{3} E_{2}^{T} E_{2}-e^{-w d} Q
\end{array}\right]+\left[\begin{array}{cc}
\Omega_{4} & 0 \\
0 & 0
\end{array}\right]<0 .
$$

Since

$$
\begin{aligned}
\Omega_{1}= & A^{T} P+P A+K^{T} B^{T} P+P B K+w P+Q+\varepsilon_{11} P P+\varepsilon_{11}^{-1} Q_{1}+\varepsilon_{12}^{-1} E_{1}^{T} E_{1}+\varepsilon_{12} P D_{1} D_{1}^{T} P \\
& +\varepsilon_{14}^{-1} K^{T} E_{3}^{T} E_{3} K+\varepsilon_{14} P D_{3} D_{3}^{T} P+\varepsilon_{13}^{-1} P D_{2} D_{2}^{T} P \\
= & A^{T} P+P A+K^{T} B^{T} P+P B K+w P+Q+\varepsilon_{1} P P+\varepsilon_{1}^{-1} Q_{1}+\varepsilon_{2}^{-1} E_{1}^{T} E_{1}+\varepsilon_{2} P D_{1} D_{1}^{T} P \\
& +\varepsilon_{4}^{-1} K^{T} E_{3}^{T} E_{3} K+\varepsilon_{4} P D_{3} D_{3}^{T} P+\varepsilon_{3}^{-1} P D_{2} D_{2}^{T} P \\
= & \Omega_{4}+K^{T} B^{T} P+P B K+\varepsilon_{4}^{-1} K^{T} E_{3}^{T} E_{3} K+\varepsilon_{4} P D_{3} D_{3}^{T} P=\Omega_{4}+\Omega_{3},
\end{aligned}
$$

(3.15) implies that (3.1) holds. According to Theorem 3.1, the system (2.4) is exponentially stable, and

$$
\|x(t)\| \leq\|\phi\| \sqrt{\frac{\lambda_{\max }(P)+d \lambda_{\max }(Q)}{\lambda_{\min }(P)}} e^{-\frac{w(t-\tau)}{2}}, \quad \forall t>0,
$$

which completes the proof.

The following theorem can be directly deduced from Theorem 3.1 by applying Lemma 2.3. 
Theorem 3.2 If there exist positive definite matrices $P>0, Q>0$ and scalar constants $\varepsilon_{i j}>0(i=1,2, j=1,2,3), \varepsilon_{14}>0, w>0$, such that the following LMIs hold:

$$
\begin{gathered}
\left(\begin{array}{cccccc}
\bar{\Omega}_{1} & P A_{d} & P & P D_{1} & P D_{2} & P D_{3} \\
* & \varepsilon_{11} Q_{2}+\varepsilon_{13} E_{2}^{T} E_{2}-e^{-w d} Q & 0 & 0 & 0 & 0 \\
* & * & -\varepsilon_{11} I & 0 & 0 & 0 \\
* & * & * & -\varepsilon_{12} I & 0 & 0 \\
* & * & * & * & -\varepsilon_{13} I & 0 \\
* & * & * & * & * & -\varepsilon_{14} I
\end{array}\right)<0, \\
\left(\begin{array}{ccccc}
\bar{\Omega}_{2} & P A_{d} & P & P D_{1} & P D_{2} \\
* & \varepsilon_{21} Q_{2}+\varepsilon_{23} E_{2}^{T} E_{2}-e^{-w d} Q & 0 & 0 & 0 \\
* & * & -\varepsilon_{21} I & 0 & 0 \\
* & * & * & -\varepsilon_{22} I & 0 \\
* & * & * & * & -\varepsilon_{23} I
\end{array}\right)<0
\end{gathered}
$$

where

$$
\bar{\Omega}_{1}=A^{T} P+P A+K^{T} B^{T} P+P B K+w P+Q+\varepsilon_{11} Q_{1}+\varepsilon_{12} E_{1}^{T} E_{1}+\varepsilon_{14} K^{T} E_{3}^{T} E_{3} K,
$$

and

$$
\bar{\Omega}_{2}=A^{T} P+P A+w P+Q+\varepsilon_{21} Q_{1}+\varepsilon_{22} E_{1}^{T} E_{1},
$$

then the system (2.4) is exponentially stable. Moreover, the solution $x(t)$ satisfies the condition

$$
\|x(t)\| \leq\|\phi\| \sqrt{\frac{\lambda_{\max }(P)+d \lambda_{\max }(Q)}{\lambda_{\min }(P)}} e^{-\frac{w(t-\tau)}{2}}, \quad \forall t>0 .
$$

Consider the following time-delay systems:

$$
\begin{aligned}
& \dot{x}(t)=A x(t)+A_{d} x(t-d)+B u(t)+f(x(t), x(t-d)), \\
& x(t)=\phi(t), \quad t \in[-d, 0],
\end{aligned}
$$

where $x \in R^{n}$ is a state vector, and $u \in R^{m}$ is the external input of the system (3.16). $f$ : $R^{n} \rightarrow R^{n}$ is a continuous nonlinear function satisfying $f(0,0)=0$, and there exist positive definite matrices $Q_{1}, Q_{2}$ such that $\|f(x(t), x(t-d))\|^{2} \leq x^{T}(t) Q_{1} x(t)+x^{T}(t-d) Q_{2} x(t-d)$ for $x(t), x(t-d) \in R^{n}$.

With the control law (2.3), the system (3.16) can be rewritten as

$$
\begin{cases}\dot{x}(t)=A x(t)+A_{d} x(t-d)+B K x(t)+f(x(t), x(t-d)), & n T \leq t<n T+\tau, \\ \dot{x}(t)=A x(t)+A_{d} x(t-d)+f(x(t), x(t-d)), & n T+\tau \leq t<(n+1) T .\end{cases}
$$

From Corollary 3.1, the following corollary can be immediately obtained. 
Corollary 3.2 If there exist positive definite matrices $P>0, Q>0$, and scalar constants $\varepsilon_{1}>0, w>0$, such that the following LMIs hold:

$$
\begin{aligned}
& {\left[\begin{array}{cc}
K^{T} B^{T} P+P B K & P A_{d} \\
A_{d}^{T} P & \varepsilon_{1}^{-1} Q_{2}-e^{-w d} Q
\end{array}\right]<0,} \\
& {\left[\begin{array}{ccc}
A^{T} P+P A+w P+Q+\varepsilon_{1}^{-1} Q_{1} & P A_{d} & P \\
A_{d}^{T} P & \varepsilon_{1}^{-1} Q_{2}-e^{-w d} Q & 0 \\
P & 0 & -\varepsilon_{1}^{-1} I
\end{array}\right]<0,}
\end{aligned}
$$

then the system (3.17) is exponentially stable. Moreover, the solution $x(t)$ satisfies the condition

$$
\|x(t)\| \leq\|\phi\| \sqrt{\frac{\lambda_{\max }(P)+d \lambda_{\max }(Q)}{\lambda_{\min }(P)}} e^{-\frac{w(t-\tau)}{2}}, \quad \forall t>0 .
$$

\section{Numerical examples}

The following numerical examples are presented to illustrate the usefulness of the proposed theoretical results in Section 3.

Example 1 Consider the following uncertain system:

$$
\begin{aligned}
\dot{x}(t)= & (A+\Delta A(t)) x(t)+\left(A_{d}+\Delta A_{d}(t)\right) x(t-d) \\
& +(B+\Delta B(t)) u(t)+f(x(t), x(t-d)), \\
x(t)= & \phi(t), \quad t \in[-d, 0],
\end{aligned}
$$

where

$$
\begin{aligned}
& A=\left[\begin{array}{ll}
-5 & -4 \\
0.1 & -7
\end{array}\right], \quad A_{d}=\left[\begin{array}{cc}
0 & 0 \\
-0.1 & -0.2
\end{array}\right], \quad B=\left[\begin{array}{ll}
1 & 0 \\
2 & 1
\end{array}\right], \\
& f(x(t), x(t-d))=\left(\begin{array}{c}
2 x_{1}(t) \sin x_{1}(t) \\
0.5 \sin x_{2}(t-d)
\end{array}\right), \quad d=2,
\end{aligned}
$$

and the uncertainties satisfy $(2.2)$ with

$$
\begin{array}{ll}
D_{1}=\left[\begin{array}{ll}
-0.1 & 0
\end{array}\right]^{T}, \quad D_{2}=\left[\begin{array}{ll}
0 & 0.1
\end{array}\right]^{T}, \quad D_{3}=\left[\begin{array}{ll}
0.1 & -0.1
\end{array}\right]^{T}, \\
E_{1}=\left[\begin{array}{ll}
0.1 & 0.1
\end{array}\right], \quad E_{2}=\left[\begin{array}{ll}
-0.1 & -0.1
\end{array}\right], \quad E_{3}=\left[\begin{array}{ll}
-0.1 & 0.1
\end{array}\right] .
\end{array}
$$

It is easy to see that the nonlinearity of the system satisfies

$$
\begin{aligned}
\|f(x(t), x(t-d))\|^{2} & =\left(2 x_{1}(t) \sin x_{1}(t)\right)^{2}+0.25 \sin ^{2} x_{2}(t-d) \\
& \leq 4 x_{1}^{2}(t)+0.25 x_{2}^{2}(t-d) .
\end{aligned}
$$

Then there exist positive definite matrices

$$
Q_{1}=\left[\begin{array}{cc}
4 & 0 \\
0 & 0.1
\end{array}\right], \quad Q_{2}=\left[\begin{array}{cc}
0.1 & 0 \\
0 & 0.25
\end{array}\right],
$$


such that

$$
\|f(x(t), x(t-d))\|^{2} \leq x^{T}(t) Q_{1} x(t)+x^{T}(t-d) Q_{2} x(t-d), \quad \text { for } x(t), x(t-d) \in R^{n} .
$$

Let $\varepsilon_{1}=\varepsilon_{2}=\varepsilon_{3}=\varepsilon_{4}=1, w=0.5$ and $K=\left[\begin{array}{cc}-15 & 8 \\ 30 & -30\end{array}\right]$ for convenience. Solving the inequalities (3.11) and (3.12), we get

$$
P=\left[\begin{array}{cc}
0.7927 & -0.3049 \\
-0.3049 & 0.4648
\end{array}\right], \quad Q=\left[\begin{array}{cc}
2.0193 & 0.0837 \\
0.0837 & 2.6127
\end{array}\right]
$$

Thus, according to Corollary 3.1, the system (4.1) is exponentially stabilizable with

$$
u(t)= \begin{cases}{\left[\begin{array}{cc}
-15 & 8 \\
30 & -30
\end{array}\right] x(t),} & n T \leq t<n T+\tau, \\
0, & n T+\tau \leq t<(n+1) T .\end{cases}
$$

Example 2 Consider the following time-delay system:

$$
\begin{aligned}
& \dot{x}(t)=A x(t)+A_{d} x(t-d)+B u(t)+f(x(t), x(t-d)), \\
& x(t)=\phi(t), \quad t \in[-d, 0],
\end{aligned}
$$

where

$$
\begin{aligned}
& f(x(t), x(t-d))=\left[\begin{array}{c}
-\frac{1}{\sqrt{2}}\left(\cos \left(x_{1}(t)\right)\right) x_{1}(t)+\sqrt{2}\left(\sin \left(x_{1}(t)\right)\right) x_{2}(t-d) \\
\frac{1}{\sqrt{2}}\left(\sin \left(x_{1}(t)\right)\right) x_{2}(t)-\sqrt{2}\left(\cos \left(x_{1}(t)\right)\right) x_{1}(t-d)
\end{array}\right], \\
& A=\left[\begin{array}{cc}
-5 & -4 \\
4 & -5
\end{array}\right], \quad A_{d}=\left[\begin{array}{cc}
0 & 0 \\
0.1 & 0.3
\end{array}\right], \\
& B=\left[\begin{array}{ll}
1 & 0 \\
1 & 2
\end{array}\right], \quad K=\left[\begin{array}{cc}
-3 & 1 \\
1 & -1
\end{array}\right], \quad d=2 .
\end{aligned}
$$

We can verify that

$$
\|f(x(t), x(t-d))\|^{2} \leq x^{T}(t) Q_{1} x(t)+x^{T}(t-d) Q_{2} x(t-d)
$$

where

$$
Q_{1}=\left[\begin{array}{ll}
1 & 0 \\
0 & 1
\end{array}\right], \quad Q_{2}=\left[\begin{array}{ll}
2 & 0 \\
0 & 2
\end{array}\right] .
$$

For $\varepsilon_{1}=1, w=0.5$, it is easy verify that

$$
P=\left[\begin{array}{cc}
1.5 & 0 \\
0 & 1.5
\end{array}\right], \quad Q=\left[\begin{array}{cc}
4 e & 0 \\
0 & 4 e
\end{array}\right],
$$

satisfy (3.18) and (3.19). 
Hence, according to Corollary 3.2, the system (4.2) is exponentially stabilizable with

$$
u(t)= \begin{cases}{\left[\begin{array}{cc}
-3 & 1 \\
1 & -1
\end{array}\right] x(t),} & n T \leq t<n T+\tau, \\
0, & n T+\tau \leq t<(n+1) T .\end{cases}
$$

\section{Conclusions}

In this brief, we deal with the exponential stabilization problem for a class of uncertain nonlinear systems with state delay by means of periodically intermittent control. Based on the Lyapunov-Krasovskii functional approach, several stability criteria have been presented in terms of a set of matrix inequalities. Moreover, the control method is extended to a class of nonlinear systems with time delay. A new sufficient condition, which guarantees the closed-loop system is exponentially stable, is presented. Finally, two numerical examples are provided to show the high performance of the proposed approach.

Competing interests

The authors declare that they have no competing interests.

Authors' contributions

YD carried out the main part of this manuscript. JL participated in the discussion and gave the examples. All authors read and approved the final manuscript.

\section{Author details}

${ }^{1}$ School of Science, Tianjin Polytechnic University, Tianjin, 300387, China. ${ }^{2}$ Binhai School of Foreign Affairs, Tianjin Foreign Studies University, Tianjin, 300270, China.

\section{Acknowledgements}

This work was supported by the Natural Science Foundation of Tianjin under Grant 11 JCYBJC06800.

Received: 20 June 2012 Accepted: 2 October 2012 Published: 17 October 2012

\section{References}

1. Dong, Y, Mei, S, Wang, X: Novel stability criteria of nonlinear uncertain systems with time-varying delay. Abstr. Appl. Anal. 2011, Article ID 969674 (2011). doi:10.1155/2011/969674

2. Phat, VN, Hein, LV: An application of Razumikhin theorem to exponential stability for linear non-autonomous systems with time-varying delay. Appl. Math. Lett. 22, 1412-1417 (2009)

3. Dong, Y, Liu, J, Mei, S, Li, M: Stabilization for switched nonlinear time-delay systems. Nonlinear Anal. Hybrid Syst. 5 , 78-88 (2011)

4. Bourkas, E, Lin, Z: Deterministic and Stochastic Time Delay systems. Birkhäuser, Boston (2002)

5. Diblik, J, Khusainov, D, Khusarenko, O: Representation of the solution for linear system of delay equations with distributed parameters. Nonlinear Dyn. Syst. Theory 13(2), 251-268 (2012)

6. Khusainov, DY, Diblik, J, Bavstinec, J, Ryvolova, A: Interval stability of linear systems neutral type. Vychisl. Prikl. Mat. 3(106), 148-160 (2011) (in Russian)

7. Dong, Y, Wang, X, Mei, S, Li, W: Exponential stabilization of nonlinear uncertain systems with time-varying delay. J. Eng. Math. 77, 225-237 (2012)

8. Shatyrko, AV, Khusainov, DY, Diblik, J, Bavstinec, J, Ryvolova, A: Estimates of perturbation of nonlinear indirect interval control system of neutral type. J. Autom. Inf. Sci. 43, 13-28 (2011)

9. Shatyrko, AV, Khusainov, DY: Absolute interval stability of indirect regulating systems of neutral type. J. Autom. Inf. Sci. 42, 43-54 (2010)

10. Shatyrko, AV, Khusainov, DY: Investigation of absolute stability of nonlinear systems of special kind with aftereffect by Lyapunov functions method. J. Autom. Inf. Sci. 43, 61-75 (2011)

11. Shatyrko, AV, Khusainov, DY: Absolute stability conditions construction of non-direct regulator systems by Lyapunov-Krasovskiy functional. Bull. Univ. Kiev, Ser. Phys. Math. 4, 145-152 (2009) (in Ukrainian)

12. Yue, D: Robust stabilization of uncertain systems with unknown input delay. Automatica 40, 331-336 (2004)

13. Diblik, J, Khusainov, D, Lukavcova, J, Raccuvzivckova, M: Control of oscillating systems with a single delay. Adv. Differ. Equ. 2010, Article ID 108218 (2010). doi:10.1155/2010/108218

14. Diblik, J, Khusainov, DY, Grytsay, IV, Šmarda, Z: Stability of nonlinear autonomous quadratic discrete systems in the critical case. Discrete Dyn. Nat. Soc. 2010, Article ID 539087 (2010). doi:10.1155/2010/539087

15. Bavstinec, J, Diblik, J, Khusainov, DY, Ryvolova, A: Exponential stability and estimation of solutions of linear differential systems of neutral type with constant coefficients. Bound. Value Probl. 2010, Article ID 956121 (2010). doi:10.1155/2010/956121

16. Diblik, J, Khusainov, DY, Grytsay, IV: Stability investigation of nonlinear quadratic discrete dynamics systems in the critical case. J. Phys. Conf. Ser. 96(1), Article ID 012042 (2008)

17. Shatyrko, AV, Khusainov, DY: Interval stability investigations of differential regulator systems with time delay by Lyapunov-Krasovskiy functional. Bull. Univ. Kiev, Ser. Phys. Math. 3, 212-221 (2009) (in Ukrainian) 
18. Dey, R, Ray, G, Ghosh, S, Rakshit, A: Stability analysis for continuous system with additive time-varying delay: a less conservative result. Appl. Math. Comput. 215, 3740-3745 (2010)

19. Khusainov, DY, Kojametov, AT, Shatyrko, AV: Interval stability of regulation systems with delay. Bull. Univ. Kiev, Ser. Phys. Math. 5, 73-88 (2001) (in Ukrainian)

20. Dzhalladova, IA, Bastinec, J, Diblik, J, Khusainov, DY: Estimates of exponential stability for solutions of stochastic control systems with delay. Abstr. Appl. Anal. 2011, Article ID 920412 (2011). doi:10.1155/2011/920412

21. Dong, Y, Mei, S: Global asymptotic stabilization of non-linear systems. Int. J. Control 82, 279-286 (2009)

22. Dong, Y, Fan, J, Mei, S: Quadratic stabilization of switched nonlinear systems. Sci. China Ser. F: Inf. Sci. 52, 999-1006 (2009)

23. Lu, JG, Hill, DJ: Impulsive synchronization of chaotic Lur'e systems by linear static measurement feedback: an LMI approach. IEEE Trans. Circuits Syst. II, Express Briefs 54, 710-714 (2007)

24. Chen, C, Ding, Z, Feng, G, Guan, X: Output regulation of discrete-time nonlinear systems based on T-S fuzzy model. In: 27th Chinese Control Conference, pp. 746-750 (2008)

25. Montgomery, TL, Frey, JW, Norris, WB: Intermittent control systems. Environ. Sci. Technol. 9, 528-532 (1975)

26. Zochowski, M: Intermittent dynamical control. Physica D, Nonlinear Phenom. 145, 181-190 (2000)

27. Li, CD, Liao, XF, Huang, TW: Exponential stabilization of chaotic systems with delay by periodically intermittent control. Chaos 17, Article ID 013103 (2007)

28. Huang, J, Li, C, Han, Q: Quasi-synchronization of chaotic neural networks with parameter mismatch by periodically intermittent control. In: World Congress on Computer Science and Information Engineering, vol. 6, pp. 485-489 (2009)

29. Wang, TY, Xie, LH, Xie, LH, De Souz, CE: Robust control of a class of uncertain nonlinear systems. Syst. Control Lett. 19, 139-149 (1992)

30. Cao, Y, Sun, Y, Cheng, C: Delay-dependent robust stabilization of uncertain systems with multiple state delays. IEEE Trans. Autom. Control 43, 1608-1612 (1998)

31. Kwon, OM, Park, JH: Exponential stability of uncertain dynamic systems including state delay. Appl. Math. Lett. 19, 901-907 (2006)

doi:10.1186/1687-1847-2012-180

Cite this article as: Dong and Liu: Exponential stabilization of uncertain nonlinear time-delay systems. Advances in Difference Equations 2012 2012:180.

\section{Submit your manuscript to a SpringerOpen ${ }^{\circ}$ journal and benefit from:}

- Convenient online submission

Rigorous peer review

- Immediate publication on acceptance

- Open access: articles freely available online

- High visibility within the field

- Retaining the copyright to your article 